\title{
Decision-making in semi-democratic contexts
}

\author{
Fiorenzo FRANCESCHINI \\ Department of Management and Production Engineering - DIGEP, Politecnico di Torino, \\ Italy \\ José Luis GARCÍA-LAPRESTA
}

PRESAD Research Group, BORDA Research Unit, IMUVA, Departamento de Economía Aplicada, Universidad de Valladolid, Spain

\begin{abstract}
A general problem, which may concern practical contexts of different nature, is to aggregate multi-experts rankings on a set of alternatives into a single fused ranking. Aggregation should also take into account the experts' importance, which may not necessarily be the same for all of them. We synthetically define this context as semi-democratic. The main aim of the paper is the analysis of the possible semi-democratic paradigms that can be conceived when the experts' importance is not the same: (i) the importance is described by means of a weighting vector; (ii) the importance is expressed by a weak order on the set of experts; (iii) the importance is described by a weak order on the set of experts with additional information on the ordinal proximities among them. The three paradigms can be applied in different decision-making situations, where some experts perform multiple assignments. In this paper various situations are discussed and analyzed in detail. A series of examples, in the field of interior design of a new car, will complement the description.
\end{abstract}

Keywords: group decision-making; semi-democratic decisions; qualitative scales; ordinal proximity measures; preferences; fusion techniques; aggregation.

\section{Introduction}

A general problem, which may concern practical contexts of different nature, is to aggregate multi-experts rankings on a set of alternatives into a single fused ranking.

Consider Table 1 , where $m$ decision-making experts ${ }^{1}$ formulate preference

Email addresses: fiorenzo.franceschini@polito.it (Fiorenzo FRANCESCHINI), lapresta@eco.uva.es (José Luis GARCÍA-LAPRESTA)

${ }^{1} \mathrm{By}$ a decision-making expert we will refer to an abstract entity able to provide a decision: human beings, individual criteria in a multi-criteria decision process, software based intelligent agents on the Internet, etc.

Preprint submitted to Information Fusion

April 27, 2019 
rankings among $n$ alternatives of interest $\left(x_{1}, x_{2}, x_{3}, x_{4}\right.$, etc.). Each ranking allows statements like $x_{1} \succ x_{2}, x_{1} \sim x_{2}$, where symbols $\succ$ and $\sim$ mean "strictly preferred to" and "indifferent to", respectively. The objective is to aggregate the $m$ experts' rankings into a single fused one, which should reflect them as much as possible, even in the presence of divergent preferences. For this reason, the fused ranking can also be defined as consensus or compromise ranking (see Cook [10] and Herrera-Viedma et al. [25]). Aggregation should also take into account the experts' importance, which is not necessarily equal for all of them.

\begin{tabular}{ccc|c} 
& Inputs & Output \\
\hline Experts & Opinions & Experts' importance & Social fused ranking \\
$e_{1}$ & $x_{2} \succ\left(x_{1} \sim x_{3}\right) \succ x_{4}$ & weights & $x_{2} \succ\left(x_{1} \sim x_{3}\right) \succ x_{4}$ \\
$e_{2}$ & $x_{3} \succ x_{2} \succ\left(x_{1} \sim x_{4}\right)$ & hierarchy & \\
$\ldots$ & $\ldots$ & hierarchy with ordinal \\
& proximity measures & \\
$e_{m}$ & $x_{4} \succ x_{1} \succ x_{2} \succ x_{3}$ & &
\end{tabular}

Table 1: Aggregation of multi-expert preference rankings into a single fused ranking.

This decision-making problem is very diffused in a variety of real-life contexts, ranging from multi-criteria decision-aiding/making to social choice theory (see Arrow and Raynaud [3] and Greco et al. [23]). Two of the reasons for this diffusion are that (i) preference rankings are probably the most intuitive and effective way to represent preference judgments of alternatives, and (ii) they do not require a common reference scale - neither numeric, linguistic or ordinal to be shared by the interacting agents (see Yager [45] and Chen et al. [8]).

The scientific literature includes a large number of aggregation models, which have been analyzed extensively from the perspective of different axioms and properties (see Arrow [2], Fishburn [13], Saari [36], Cook [10] and Nurmi [34], among others).

A long and lively debate has involved many scientists on the effects that the Arrow's theorem can induce on practical decisions (see Arrow [2], Arrow and Raynaud [3], Franssen [19], See and Lewis [38], Keeney [28], Ladha et al. [29] and McComb et al. [31], among others). Some researchers have demonstrated the effectiveness of specific aggregation models, even though they do not satisfy some of the basic properties related to the Arrow's theorem. For instance, Dym et al. [11] showed that, although the Borda aggregation model may not satisfy the Independence of Irrelevant Alternatives condition, this event rarely affect the most preferred alternatives. They concluded that Arrow's theorem poses a considerable theoretical problem, but the practical implications are not so worrisome.

Additional research has been carried out by See and Lewis [38], proposing a structured approach to avoid severe theoretical conflicts. Jacobs et al. [27] 
recognized several additional issues related to the uncertainty, comparability and measurability concerned with aggregation models, both in the aggregation of preferences and performances.

Franceschini and Maisano $[16,17]$ addressed the problem of the coherence between decision agent preferences and collective preference ranking. Other researchers focused their studies on proposing new approaches to manage linguistic distribution assessment in multi-attribute group decision making (see Keeney [28], de Andrés et al. [1], García-Lapresta and Pérez-Román [22], Yu et al. [46], Zhang et al. [49], Wu et al. [43] and Ureña et al. [40], among others).

The literature is also rich in many practical applications in various fields. As an example, a small review is shown in Table 2 (see Greco et al. [23], Önüt et al. [35], Yager [25], Griffin and Hauser [24], Franceschini et al. [14], Colomer [9], Saari [36] and Fishburn [13]).

The main aim of the paper is the analysis of the possible paradigms that can be conceived when the experts' importance is not the same. We synthetically define this context as semi-democratic. The term semi-democracy is used to refer to a context that shares both democratic and authoritarian features (see Møller and Skaaning [33]). In this specific framework the term is used to highlight that all experts participate (democracy) to the collective fused ranking, although they may have a different weight in the decision (semi or partial democracy). The modelling of these decision-making problems has only been partially explored in the literature. The complexity of the problem is due to the difficulty of modeling the different degree of importance of the decision experts.

In some cases the numerical weight associated with each single expert is known, while in other cases only the expert hierarchy (but not their relative weights) is known. In some other situations it is possible to know, in addition to the expert hierarchy, also the proximities between the hierarchy levels.

Most of the papers in the literature focus their attention on decision problems where the weights associated with the individual experts are known (see Arrow and Raynaud [3] and Greco et al. [23]); other papers concentrate on the technique for determining these weights (see Yue [47], Dubois et al. [12], Zhang and Guo [48], Mishra and Rani [32] and Hu et al. [26], among others). However, when we move on to less structured problems, where the hierarchy level between decision makers is expressed by more nuanced information i.e., only the hierarchical level of experts is known) or by a hierarchical proximity (i.e., only the hierarchical proximity of experts is known), the literature offers only few approaches for tackling this problem (see Yager [45]).

By this paper we wish to provide a structured conceptual framework on the state of the art and on the potential future research areas for decision-making problems in semi-democratic contexts. In this manuscript various situations will be discussed and analyzed in detail. A series of examples, inspired by a real application in the field of interior design of a new car, will complement the description.

The rest of the paper is organized as follows. Section 2 introduces Borda scores and ordinal proximity measures. Section 3 includes the paradigms considered in the paper to categorize semi-democratic contexts. Section 4 contains 
Field

Multicriteria decision

aiding/making

Agents

Alternatives

Qualitative/quantitative Alternative locations criteria

Multicriteria decision aiding/making

\section{Internet}

Quality management

Voting theory
Qualitative/quantitative Technology selection criteria

Different types of in-

formation concerning

Data displayed on Inter-

net sites

the user

Questionnaire/interview Customer requirements respondents

Voters
Candidates in an election
Problem description

Determination of the best location where to install a new manufacturing plant on the basis of several criteria such as $\mathrm{road} /$ railway infrastructure, electrical supply, labour cost, etc. (see Greco et al. [23])

Machine tool selection (see Önüt et al. [35])

Intelligent customization of data displayed on Internet sites, based on several types of information such as user's country, websites visited previously, apps downloaded, etc. (see Yager [44])

Synthesis of customer requirements, which are evaluated by a sample of questionnaire/interview respondents (see Griffin and Hauser [24] and Franceschini et al. [14])

Searching a reasonable mechanism for aggregating the opinions expressed by several voters on the candidates, in order to determine a winner or to rank all candidates in order of preference (see Colomer [9], Fishburn [13] and Saari [36])

Table 2: Examples of practical applications of the problem of interest. 
a case study. Section 5 includes some concluding remarks.

\section{Preliminaries}

Let $E=\left\{e_{1}, \ldots, e_{m}\right\}$ be a set of experts. With $W(E)$ we denote the set of weak orders (or complete preorders) on $E$. Given $S \in W(E)$, with $\succ$ and $\sim$ we denote the asymmetric and the symmetric parts of $S$, respectively. Given a set $Y$, with $\# Y$ we denote the cardinality of $Y$.

Definition 1. Given $S \in W(E)$, let $B: E \longrightarrow\{0,0.5,1, \ldots, m-1.5, m-1\}$ be the mapping that assigns the Borda score of each expert $e_{i} \in E$, defined as

$$
B\left(e_{i}\right)=\#\left\{e_{j} \in E \mid e_{i} \succ e_{j}\right\}+\frac{1}{2} \#\left\{e_{j} \in\left(E \backslash\left\{e_{i}\right\}\right) \mid e_{j} \sim e_{i}\right\} .
$$

Example 1. Consider $S \in W\left(\left\{e_{1}, \ldots, e_{5}\right\}\right)$ given by

$$
\begin{gathered}
S \\
\hline e_{1} \\
e_{2} e_{3} \\
e_{4} e_{5}
\end{gathered}
$$

i.e., $e_{1} \succ\left(e_{2} \sim e_{3}\right) \succ\left(e_{4} \sim e_{5}\right)$.

Then, we have $B\left(e_{1}\right)=4, B\left(e_{2}\right)=B\left(e_{3}\right)=2.5$ and $B\left(e_{4}\right)=B\left(e_{5}\right)=0.5$.

We now recall the notion of ordinal proximity measure, introduced by GarcíaLapresta and Pérez-Román [22]. An ordinal proximity measure is a mapping that assigns an ordinal degree of proximity to each pair of linguistic terms of an ordered qualitative scale (OQS) $\mathcal{L}=\left\{l_{1}, \ldots, l_{g}\right\}$, with $l_{1}<\cdots<l_{g}$ and $g \geq 3$. The mentioned ordinal degrees of proximity belong to a linear order $\Delta=\left\{\delta_{1}, \ldots, \delta_{h}\right\}$, with $\delta_{1} \succ \cdots \succ \delta_{h}$ and $h \geq 3$, being $\delta_{1}$ and $\delta_{h}$ the maximum and minimum degrees of proximity, respectively. It is important noticing that the elements of $\Delta$ are not numbers. In fact, they are only abstract objects representing different degrees of proximity.

Definition 2. ([22]) An ordinal proximity measure $(\boldsymbol{O P M})$ on $\mathcal{L}$ with values in $\Delta$ is a mapping $\pi: \mathcal{L}^{2} \longrightarrow \Delta$, where $\pi\left(l_{r}, l_{s}\right)=\pi_{r s}$ represents the degree of proximity between $l_{r}$ and $l_{s}$, satisfying the following conditions:

1. Exhaustiveness: For every $\delta \in \Delta$, there exist $l_{r}, l_{s} \in \mathcal{L}$ such that $\delta=\pi_{r s}$.

2. Symmetry: $\pi_{s r}=\pi_{r s}$, for all $r, s \in\{1, \ldots, g\}$.

3. Maximum proximity: $\pi_{r s}=\delta_{1} \Leftrightarrow r=s$, for all $r, s \in\{1, \ldots, g\}$.

4. Monotonicity: $\pi_{r s} \succ \pi_{r t}$ and $\pi_{s t} \succ \pi_{r t}$, for all $r, s, t \in\{1, \ldots, g\}$ such that $r<s<t$. 
Every OPM $\pi: \mathcal{L}^{2} \longrightarrow \Delta$ can be represented by a $g \times g$ symmetric matrix with coefficients in $\Delta$, where the elements in the main diagonal are $\pi_{r r}=\delta_{1}$, $r=1, \ldots, g$ :

$$
\left(\begin{array}{ccccc}
\pi_{11} & \cdots & \pi_{1 s} & \cdots & \pi_{1 g} \\
\cdots & \cdots & \cdots & \cdots & \cdots \\
\pi_{r 1} & \cdots & \pi_{r s} & \cdots & \pi_{r g} \\
\cdots & \cdots & \cdots & \cdots & \cdots \\
\pi_{g 1} & \cdots & \pi_{g s} & \cdots & \pi_{g g}
\end{array}\right) .
$$

This matrix is called the proximity matrix associated with $\pi$.

Some procedures for generating OPMs in an OQS are introduced by GarcíaLapresta et al. [21].

\section{Paradigms to categorize semi-democratic contexts}

Let $E=\left\{e_{1}, \ldots, e_{m}\right\}$ be a set of experts that show their preferences on a set of alternatives $X=\left\{x_{1}, \ldots, x_{n}\right\}$ through a profile of weak orders $\left(R_{1}, \ldots, R_{m}\right) \in W(X)^{m}$.

The aim is to generate a social weak order $R^{*} \in W(X)$ representing individual preferences taking into account that the importance of experts may be different.

According to the content of Table 3 , we can identify three potential paradigms to categorize the concept of semi-democratic context for a set of experts:

1. A numerical weight $w_{i} \in[0,1]$ is assigned to each expert $e_{i} \in E$.

2. The set of experts is categorized by a hierarchy, i.e., a weak order on the set of experts, $S \in W(E)$.

3. The set of experts is categorized by a graduated hierarchy within an OQS equipped with an OPM, $v_{1}, \ldots, v_{m} \in \mathcal{L}$.

\begin{tabular}{ccl|c} 
& Inputs & Output \\
\hline Experts & Opinions & Experts' importance & Social fused ranking \\
$e_{1}$ & $R_{1} \in W(X)$ & $w_{1}, \ldots, w_{m} \in[0,1]$ & $R^{*} \in W(X)$ \\
$e_{2}$ & $R_{2} \in W(X)$ & $S \in W(E)$ & \\
$\ldots$ & $\ldots$ & $v_{1}, \ldots, v_{m} \in \mathcal{L}$ & \\
$e_{m}$ & $R_{m} \in W(X)$ & &
\end{tabular}

Table 3: Aggregation of multi-expert preference rankings into a single fused ranking.

\subsection{Assigning a numerical weight to each expert}

In some contexts experts may have recognized abilities and attributes and/or privileged positions of power, represented by weights (see Dubois et al. [12], Brans and Mareschal [6] and Greco et al. [23]). 
The definition of the experts' weights can be a very delicate issue. In some settings, the weight of an expert may be well defined; for example, the Gross National Product (GNP) or population size of a country represented by the member on an international committee can immediately be used as weights. In many other situations the definition of the weights is controversial, because there are no indisputable criteria that can be used for this operation. Weights are often imposed by decision-makers, according to political strategies (see Wang et al. [42]). For example, the scientific committee of a competitive examination for promotion of Faculty members may decide that scientific publications will account for $30 \%$ of the total performance, the international projects for $25 \%$, the teaching activity for $35 \%$, etc.

The literature includes several techniques for the quantification of weights. For example, the AHP procedure uses the eigenvector method to derive a weight vector relating to experts (see Saaty [37]), while the method proposed by Martel and Ben Khélifa [30] determines the so-called "relative importance coefficient" of each expert, based on the combination of subjective and objective components.

More specifically, the importance of experts is directly reported by means of a weighting vector $\boldsymbol{w}=\left(w_{1}, \ldots, w_{m}\right) \in[0,1]^{m}$ such that $w_{1}+\cdots+w_{m}=1$ and $100 w_{i} \in \mathbb{N}$, for every $i \in\{1, \ldots m\}$.

The weighting scheme should follow the replication proposal given by GarcíaLapresta and González del Pozo [20]: the weak orders associated with the experts are replicated according to the corresponding percentages, $100 w_{1}, \ldots$, $100 w_{m}$. In practice, it should be convenient to calculate the greatest common divisor (gcd) of percentages associated with the weights, and divide each percentage by the gcd. Then, the minimum number of replications of each profile is obtained:

$$
t_{i}=\frac{100 w_{i}}{\operatorname{gcd}\left\{100 w_{1}, \ldots, 100 w_{m}\right\}}, \quad i \in\{1, \ldots, m\} .
$$

If $\left(t_{1}, \ldots, t_{m}\right) \notin \mathbb{N}^{m}$, then these numbers have to be properly rounded.

Thus, the experts' weak orders of the original profile $\left(R_{1}, \ldots, R_{m}\right) \in W(X)^{m}$ are replicated accordingly:

$$
(\overbrace{R_{1}, \ldots, R_{1}}^{t_{1}}, \ldots, \overbrace{R_{m}, \ldots, R_{m}}^{t_{m}}) \in W(X)^{t_{1}+\cdots+t_{m}} .
$$

In some settings, weights are not available or cannot be defined on cardinal scales. In these cases, the importance hierarchy of agents may be expressed by a weak order (see Yager [45]). When the expert importance prioritization is doubtful, the formulation of rankings is certainly simpler and more intuitive than the formulation of weights (see Chen et al. [8]).

\subsection{Hierarchy of experts}

In this subsection we will focus on a specific aggregation problem in which the experts' importance is expressed through a weak order. This decision-making 
context can be denominated as "ordinal semi-democratic"; the adjective "semidemocratic" indicates that agents do not necessarily have the same importance, while "ordinal" indicates that their rank is defined by a crude ranking. This makes the set of the possible solutions relatively wide, since they may range between the two extreme situations of (i) full dictatorship - in which the resulting fused ranking coincides with the preference ranking by the most important agent (dictator) - and (ii) full democracy - where the agents' preference rankings are considered as equi-important.

In spite of its practicality and adaptability to a large number of real contexts, this specific decision-making problem is almost completely ignored in the literature. Over ten years ago, Yager [45] proposed an algorithm (hereafter abbreviated as YA, which stands for Yager's Algorithm) to address this problem in a relatively simple and fast way. Unfortunately, this algorithm has two important limitations: (i) the resulting fused ranking may sometimes not reflect the preference ranking for the majority of experts (see Wang [41]) and (ii) it is only applicable to linear orders, without incomparabilities and omissions of the alternatives of interest. The paper of Franceschini et al. [18] enhances the YA in order to overcome its limitations and adapt to less stringent preference rankings.

In a formal way, the importance of experts is represented by means of a weak order $S \in W(E)$. In this situation we can operate in two ways:

1. Direct method: adopting the YA algorithm or similar variants (Yager [45], Franceschini et al. [18]; see Section 4).

2. Indirect method: we can generate an "artificial" weighting vector based, for example, on the Borda scores obtained by the experts (Definition 1),

$$
w_{i}=\frac{B\left(e_{i}\right)}{B\left(e_{1}\right)+\cdots+B\left(e_{m}\right)}, \quad i \in\{1, \ldots, m\},
$$

going back to the case analyzed in Subsection 3.1. It can be noticed that Sen [39] has already considered the Borda scores as weights of the objects in a ranking.

In the Example 1, the following weighting vector $\boldsymbol{w}=(0.4,0.25,0.25,0.05,0.05)$ is obtained.

Since $\operatorname{gcd}\left\{100 w_{1}, \ldots, 100 w_{5}\right\}=\operatorname{gcd}\{40,25,25,5,5\}=5$, then $R_{1}, R_{2}, R_{3}$, $R_{4}$ and $R_{5}$ should be replicated $40 / 5=8,25 / 5=5,25 / 5=5,5 / 5=1$ and $5 / 5=1$ times, respectively:

$$
(\overbrace{R_{1}, \ldots, R_{1}}^{8}, \overbrace{R_{2}, \ldots, R_{2}}^{5}, \overbrace{R_{3}, \ldots, R_{3}}^{5}, R_{4}, R_{5}) \in W(X)^{20} .
$$

An equivalent approach consists of directly calculate the number of replications avoiding to obtain the weighting vector and the corresponding rounding problems. 
Since $B: E \longrightarrow\{0,0.5,1, \ldots, m-1.5, m-1\}$, i.e., $B\left(e_{i}\right)$ could be not integer, consider

$$
t_{i}=\frac{2 B\left(e_{i}\right)}{\operatorname{gcd}\left\{2 B\left(e_{1}\right), \ldots, 2 B\left(e_{m}\right)\right\}}, \quad i \in\{1, \ldots, m\} .
$$

So, in Example 1 we directly obtain $t_{1}=8, t_{2}=t_{3}=5$ and $t_{4}=t_{5}=1$.

Notice that the importance of expert $e_{1}$ is $8 / 5=1.6$ times the importance of experts $e_{2}$ and $e_{3}$; the importance of expert $e_{1}$ is $8 / 1=8$ times the importance of experts $e_{4}$ and $e_{5}$; and the importance of experts $e_{2}$ and $e_{3}$ is $5 / 1=5$ times the importance of experts $e_{4}$ and $e_{5}$.

\subsection{Ordinal proximity measures}

This subsection introduces the third paradigm. In this case the experts' importance is expressed again through a ranking, with an additional measure of the proximity (proximity graduation) of the ordinal semi-democratic hierarchy.

A decision-maker evaluates the experts in an OQS $\mathcal{L}=\left\{l_{1}, \ldots, l_{g}\right\}$ equipped with an OPM $\pi: \mathcal{L}^{2} \longrightarrow \Delta=\left\{\delta_{1}, \ldots, \delta_{h}\right\}$, by assigning a linguistic term $v_{i} \in \mathcal{L}$ to each expert $e_{i} \in E$. Let $\rho: \Delta \longrightarrow \mathbb{N}$ be the mapping defined as $\rho\left(\delta_{r}\right)=r$.

A score is given to each expert $e_{i} \in E$ through the mapping $S: E \longrightarrow \mathbb{R}$ defined as

$$
S\left(e_{i}\right)=h+\rho\left(\pi\left(v_{i}, l_{1}\right)\right)-\rho\left(\pi\left(v_{i}, l_{g}\right)\right)+\sum_{v_{i}>v_{j}} \rho\left(\pi\left(v_{i}, v_{j}\right)\right)+\frac{1}{2} \sum_{\substack{v_{i}=v_{j} \\ i \neq j}} \rho\left(\pi\left(v_{i}, v_{j}\right)\right) .
$$

Since $S\left(e_{i}\right)$ could be not integer, following the same pattern that in (2), consider

$$
t_{i}=\frac{2 S\left(e_{i}\right)}{\operatorname{gcd}\left\{2 S\left(e_{1}\right), \ldots, 2 S\left(e_{m}\right)\right\}}, \quad i \in\{1, \ldots, m\} .
$$

The meaning of $S\left(e_{i}\right)$ in (3) is explained as follows. In order to all the scores $S\left(e_{1}\right), \ldots, S\left(e_{m}\right)$ be positive, $h$ points are initially assigned to each expert, just the number of ordinal degrees of proximity; $\pi\left(v_{i}, l_{1}\right)$ measures the proximity between the assessment of $e_{i}$ and the lowest possible assessment, $l_{1}$ (the lower, the better); $\pi\left(v_{i}, l_{g}\right)$ measures the proximity between the assessment of $e_{i}$ and the highest possible assessment, $l_{g}$ (now the higher, the better). Consequently, $\rho\left(\pi\left(v_{i}, l_{1}\right)\right)-\rho\left(\pi\left(v_{i}, l_{g}\right)\right)$ is the number of steps for going from $\pi\left(v_{i}, l_{1}\right)$ to $\pi\left(v_{i}, l_{g}\right)$, being this difference positive whenever the assessment of $e_{i}$ is closer to $l_{g}$ than to $l_{1}$, and negative in the opposite case.

Notice that $h+\rho\left(\pi\left(v_{i}, l_{1}\right)\right)-\rho\left(\pi\left(v_{i}, l_{g}\right)\right)$ can be considered as the absolute part of the score $S\left(e_{i}\right)$, in the sense that it does not depend on the assessments obtained for other experts.

However, the second part of $S\left(e_{i}\right)$,

$$
\sum_{v_{i}>v_{j}} \rho\left(\pi\left(v_{i}, v_{j}\right)\right)+\frac{1}{2} \sum_{\substack{v_{i}=v_{j} \\ i \neq j}} \rho\left(\pi\left(v_{i}, v_{j}\right)\right)
$$


can be considered as the relative part of the score $S\left(e_{i}\right)$, since it depends of the assessments obtained for other experts.

With $\sum_{v_{i}>v_{j}} \rho\left(\pi\left(v_{i}, v_{j}\right)\right)$ we take into account the ordinal degrees of proximity between the assessment of $e_{i}$ and those obtained by the experts that have been evaluated worse than $e_{i}$. Finally, $\sum_{\substack{v_{i}=v_{j} \\ i \neq j}} \rho\left(\pi\left(v_{i}, v_{j}\right)\right)$ is just the number of experts that share with $e_{i}$ the same assessment.

Obviously, $S\left(e_{i}\right)>S\left(e_{j}\right) \Leftrightarrow v_{i}>v_{j}$.

Remark 1. The maximum score that an expert $e_{i}$ can reach is obtained when $e_{i}$ has the highest assessment, $l_{g}$, and the rest of experts have the lowest assessment, $l_{1}$; in this case, $S\left(e_{i}\right)=h+h-1+(m-1) h=(m+1) h-1$. Conversely, the minimum score that an expert $e_{i}$ can reach is obtained when $e_{i}$ has the lowest assessment, $l_{1}$, and the rest of experts have the highest assessment, $l_{g}$; now, $S\left(e_{i}\right)=h+1-h=1$. Thus, $1 \leq S\left(e_{i}\right) \leq(m+1) h-1$.

Remark 2. The relative part of the score $S\left(e_{i}\right)$ depends on the modifications on the set of experts:

1. Consider a new expert $e_{m+1}$. Let $S^{\prime}: E \cup\left\{e_{m+1}\right\} \longrightarrow \mathbb{R}$ be the new mapping. Then, $v_{i} \geq v_{m+1} \Rightarrow S^{\prime}\left(e_{i}\right)>S\left(e_{i}\right)$.

2. If an expert $e_{k}$ is removed, let $S^{\prime}: E \backslash\left\{e_{k}\right\} \longrightarrow \mathbb{R}$ be the new mapping. Then, $v_{i} \geq v_{k} \Rightarrow S^{\prime}\left(e_{i}\right)<S\left(e_{i}\right)$.

Example 2. Let $\mathcal{L}=\left\{l_{1}, l_{2}, l_{3}, l_{4}\right\}$ be the OQS equipped with the OPM with associated proximity matrix ${ }^{2}$

$$
A_{342}=\left(\begin{array}{cccc}
\delta_{1} & \delta_{3} & \delta_{6} & \delta_{7} \\
& \delta_{1} & \delta_{4} & \delta_{5} \\
& & \delta_{1} & \delta_{2} \\
& & & \delta_{1}
\end{array}\right)
$$

that can be visualized in Fig. 1.

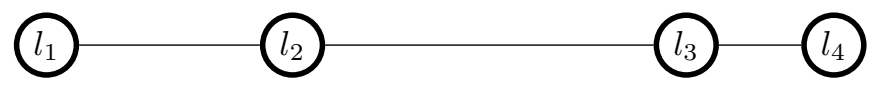

Figure 1: Ordinal proximity measure with associated matrix $A_{342}$.

The meaning of the proximity matrix $A_{342}$ is that the ordinal degrees of proximity between the linguistic terms of the OQS $\mathcal{L}$ are $\pi\left(l_{3}, l_{4}\right)=\delta_{2} \succ$ $\pi\left(l_{1}, l_{2}\right)=\delta_{3} \succ \pi\left(l_{2}, l_{3}\right)=\delta_{4} \succ \pi\left(l_{2}, l_{4}\right)=\delta_{5} \succ \pi\left(l_{1}, l_{3}\right)=\delta_{6} \succ \pi\left(l_{1}, l_{4}\right)=\delta_{7}$.

The absolute part of the score $S\left(e_{i}\right)$ is included in Table 4 for the four possible assessments that experts can obtain.

${ }^{2}$ The subindices 342 of the matrix $A_{342}$ correspond to the subindices of the $\delta$ 's appearing 


\begin{tabular}{cccl}
$v_{i}$ & $\pi\left(v_{i}, l_{1}\right)$ & $\pi\left(v_{i}, l_{g}\right)$ & $h+\rho\left(\pi\left(v_{i}, l_{1}\right)\right)-\rho\left(\pi\left(v_{i}, l_{g}\right)\right)$ \\
\hline$l_{1}$ & $\delta_{1}$ & $\delta_{7}$ & $7+1-7=1$ \\
$l_{2}$ & $\delta_{3}$ & $\delta_{5}$ & $7+3-5=4$ \\
$l_{3}$ & $\delta_{6}$ & $\delta_{2}$ & $7+6-2=11$ \\
$l_{4}$ & $\delta_{7}$ & $\delta_{1}$ & $7+7-1=13$
\end{tabular}

Table 4: Absolute part of the score $S\left(e_{i}\right)$.

Example 3. Let $\mathcal{L}=\left\{l_{1}, l_{2}, l_{3}, l_{4}\right\}$ be the OQS equipped with the OPM with associated proximity matrix

$$
A_{232}=\left(\begin{array}{cccc}
\delta_{1} & \delta_{2} & \delta_{4} & \delta_{5} \\
& \delta_{1} & \delta_{3} & \delta_{4} \\
& & \delta_{1} & \delta_{2} \\
& & & \delta_{1}
\end{array}\right)
$$

that can be visualized in Fig. 2.

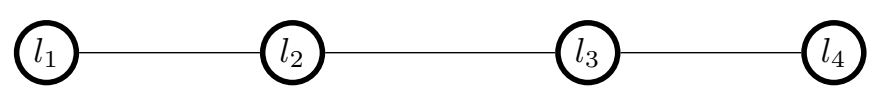

Figure 2: Ordinal proximity measure with associated matrix $A_{232}$.

We consider Example 1, where $e_{1} \succ\left(e_{2} \sim e_{3}\right) \succ\left(e_{4} \sim e_{5}\right)$, and we take into account the four possible experts' evaluations in $\mathcal{L}=\left\{l_{1}, l_{2}, l_{3}, l_{4}\right\}$ compatible with the mentioned experts' importance ranking:

1. $v_{1}=l_{4}, v_{2}=v_{3}=l_{3}$ and $v_{4}=v_{5}=l_{2}$.

After applying (3), we have $S\left(e_{1}\right)=21, S\left(e_{2}\right)=S\left(e_{3}\right)=13.5$ and $S\left(e_{4}\right)=S\left(e_{5}\right)=3.5$. Then, $t_{1}=42, t_{2}=t_{3}=27$ and $t_{4}=t_{5}=7$. Thus, $R_{1}, R_{2}, R_{3}, R_{4}$ and $R_{5}$ should be replicated $42,27,27,7$ and 7 times, respectively:

$$
(\overbrace{R_{1}, \ldots, R_{1}}^{42}, \overbrace{R_{2}, \ldots, R_{2}}^{27}, \overbrace{R_{3}, \ldots, R_{3}}^{27}, \overbrace{R_{4}, \ldots, R_{4}}^{27}, \overbrace{R_{5}, \ldots, R_{5}}^{7}) \in W(X)^{110} .
$$

Then, the importance of expert $e_{1}$ is $42 / 27=1.55$ times the importance of experts $e_{2}$ and $e_{3}$; the importance of expert $e_{1}$ is $42 / 7=6$ times the importance of experts $e_{4}$ and $e_{5}$; and the importance of experts $e_{2}$ and $e_{3}$ is $27 / 7=3.86$ times the importance of experts $e_{4}$ and $e_{5}$.

2. $v_{1}=l_{4}, v_{2}=v_{3}=l_{3}$ and $v_{4}=v_{5}=l_{1}$.

in the coefficients just over the main diagonal. We follow the same pattern in subsequent matrices. 
After applying (3), we have $S\left(e_{1}\right)=23, S\left(e_{2}\right)=S\left(e_{3}\right)=15.5$ and $S\left(e_{4}\right)=S\left(e_{5}\right)=1.5$. Then, $t_{1}=46, t_{2}=t_{3}=31$ and $t_{4}=t_{5}=3$. Thus, $R_{1}, R_{2}, R_{3}, R_{4}$ and $R_{5}$ should be replicated $46,31,31,3$ and 3 times, respectively:

$$
(\overbrace{R_{1}, \ldots, R_{1}}^{46}, \overbrace{R_{2}, \ldots, R_{2}}^{31}, \overbrace{R_{3}, \ldots, R_{3}}^{31}, \overbrace{R_{4}, \ldots, R_{4}}^{31}, \overbrace{R_{5}, \ldots, R_{5}}^{3}) \in W(X)^{114} .
$$

Then, the importance of expert $e_{1}$ is $46 / 31=1.48$ times the importance of experts $e_{2}$ and $e_{3}$; the importance of expert $e_{1}$ is $46 / 3=15.33$ times the importance of experts $e_{4}$ and $e_{5}$; and the importance of experts $e_{2}$ and $e_{3}$ is $31 / 3=10.33$ times the importance of experts $e_{4}$ and $e_{5}$.

3. $v_{1}=l_{4}, v_{2}=v_{3}=l_{2}$ and $v_{4}=v_{5}=l_{1}$.

After applying (3), we have $S\left(e_{1}\right)=27, S\left(e_{2}\right)=S\left(e_{3}\right)=7.5$ and $S\left(e_{4}\right)=$ $S\left(e_{5}\right)=1.5$. Now, $t_{1}=18, t_{2}=t_{3}=5$ and $t_{4}=t_{5}=1$. Thus, $R_{1}, R_{2}$, $R_{3}, R_{4}$ and $R_{5}$ should be replicated $18,5,5,1$ and 1 times, respectively:

$$
(\overbrace{R_{1}, \ldots, R_{1}}^{18}, \overbrace{R_{2}, \ldots, R_{2}}^{5}, \overbrace{R_{3}, \ldots, R_{3}}^{5}, R_{4}, R_{5}) \in W(X)^{30} .
$$

Then, the importance of expert $e_{1}$ is $18 / 5=3.6$ times the importance of experts $e_{2}$ and $e_{3}$; the importance of expert $e_{1}$ is $18 / 1=18$ times the importance of experts $e_{4}$ and $e_{5}$; and the importance of experts $e_{2}$ and $e_{3}$ is $5 / 1=5$ times the importance of experts $e_{4}$ and $e_{5}$.

4. $v_{1}=l_{3}, v_{2}=v_{3}=l_{2}$ and $v_{4}=v_{5}=l_{1}$.

After applying (3), we have $S\left(e_{1}\right)=21, S\left(e_{2}\right)=S\left(e_{3}\right)=7.5$ and $S\left(e_{4}\right)=$ $S\left(e_{5}\right)=1.5$. Now, $t_{1}=14, t_{2}=t_{3}=5$ and $t_{4}=t_{5}=1$. Thus, $R_{1}, R_{2}$, $R_{3}, R_{4}$ and $R_{5}$ should be replicated 14, 5, 5, 1 and 1 times, respectively:

$$
(\overbrace{R_{1}, \ldots, R_{1}}^{14}, \overbrace{R_{2}, \ldots, R_{2}}^{5}, \overbrace{R_{3}, \ldots, R_{3}}^{5}, R_{4}, R_{5}) \in W(X)^{26} .
$$

Then, the importance of expert $e_{1}$ is $14 / 5=2.8$ times the importance of experts $e_{2}$ and $e_{3}$; the importance of expert $e_{1}$ is $14 / 1=14$ times the importance of experts $e_{4}$ and $e_{5}$; and the importance of experts $e_{2}$ and $e_{3}$ is $5 / 1=5$ times the importance of experts $e_{4}$ and $e_{5}$.

Remark 3. Notice that in the procedure of Subsection 3.2, the number of replications is univocally determined by Eq. (2). However, in the procedure of Subsection 3.3 the number of replications can vary depending on the OPM considered on the OQS and the evaluations obtained by the experts. For instance, in Example 3, the OQS has 4 linguistic terms and it can be equipped 
with 51 different OPMs. Additionally, experts can be evaluated in 4 different ways compatible with the weak order $e_{1} \succ\left(e_{2} \sim e_{3}\right) \succ\left(e_{4} \sim e_{5}\right)$. Thus, 204 kinds of replications are possible.

\subsection{Aggregation}

In the paradigms presented in Section 3, a profile of weak orders on the set of alternatives $X=\left\{x_{1}, \ldots, x_{n}\right\}$ is obtained. To generate a collective weak order on $X$, it is necessary to consider an aggregation rule. A prominent class of aggregation rules is the family of scoring rules (see Chebotarev and Shamis [7]) and, particularly, the Borda rule [5].

Initially, the Borda rule was devised for linear orders (indifferences are not allowed). There are several ways to adjust the Borda rule to weak orders (see Black [4]). We follow the pattern of Eq. (1).

The Borda score of each alternative $x_{i} \in X$ is defined for every individual weak order $R_{k} \in W(X)$ of the profile $\left(R_{1}, \ldots, R_{m}\right) \in W(X)^{m}$.

Let $B_{k}: X \longrightarrow\{0,0.5,1, \ldots, n-1.5, n-1\}$ be the mapping that assigns the Borda score of each alternative $x_{i} \in X$ for $R_{k} \in W(X)$, which is defined as

$$
B_{k}\left(x_{i}\right)=\#\left\{x_{j} \in X \mid x_{i} \succ_{k} x_{j}\right\}+\frac{1}{2} \#\left\{x_{j} \in\left(X \backslash\left\{x_{i}\right\}\right) \mid x_{j} \sim_{k} x_{i}\right\} .
$$

Then, a total score is obtained for each alternative,

$$
B^{*}\left(x_{i}\right)=\sum_{k=1}^{m} B_{k}\left(x_{i}\right)
$$

and the collective weak order on $X$ generated by the Borda rule, $R^{*}$, is defined as

$$
x_{i} R^{*} x_{j} \Leftrightarrow B^{*}\left(x_{i}\right) \geq B^{*}\left(x_{j}\right)
$$

for all $x_{i}, x_{j} \in X$.

\section{Case study}

We propose an application of the above concepts in the field of interior design of a new car.

A set of interior settings of a new car are provided to a customer panel selected on the base of the fidelity to the brand and on the level of education. A sample of ten customers/experts $E=\left\{e_{1}, \ldots, e_{10}\right\}$ were encouraged to analyze five interior designs for a new car, $X=\left\{x_{1}, x_{2}, x_{3}, x_{4}, x_{5}\right\}$.

Customers were divided into four classes of importance within the OQS $\mathcal{L}=\left\{l_{1}, l_{2}, l_{3}, l_{4}\right\}$, namely $l_{1}=\mathrm{D}, l_{2}=\mathrm{C}, l_{3}=\mathrm{B}$ and $l_{4}=\mathrm{A}$, based on the two analysis dimensions: (i) the "fidelity to the brand" (number of years) and (ii) the "level of education" (e.g., bachelor, master, doctorate). 
These two dimensions may significantly influence the accuracy of the response while being relatively easy to evaluate. The two dimensions can be described through the two-dimensional map in Fig. 3.

The most important customers (in class A) are those with relatively high values in both dimensions. According to a lexicographic ranking, which favors the former dimension with respect to the latter, the second and third most important classes are respectively B and C. The least important customers (in class D) are those with relatively low values in both dimensions.

Of course, the importance ranking could be based on additional and/or substitute analysis dimensions (e.g., "age of the respondents", etc.) or different evaluation criteria.

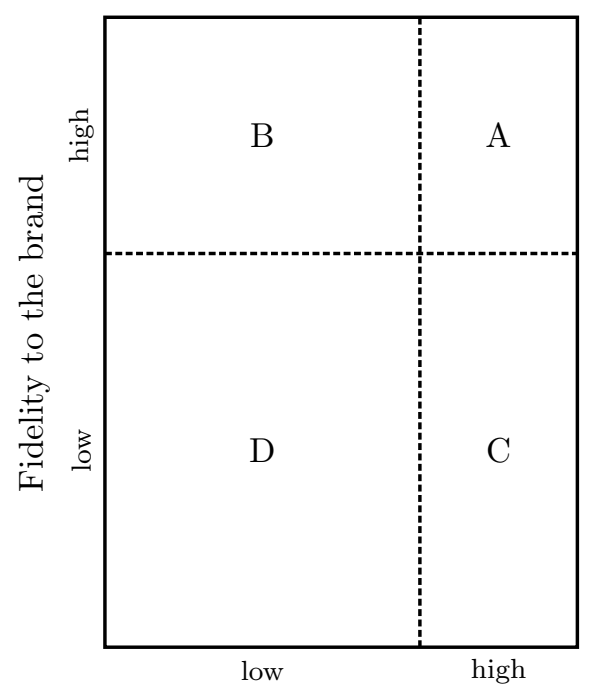

Education level of the respondent

Figure 3: Qualitative map to discriminate the importance classes of customers.

Based on the above considerations, the resulting importance ranking of customers is:

$$
\begin{aligned}
e_{1} e_{7} & \mapsto \mathrm{A} \\
e_{2} e_{4} & \mapsto \mathrm{B} \\
e_{3} e_{5} e_{10} & \mapsto \mathrm{C} \\
e_{6} e_{8} e_{9} & \mapsto \mathrm{D}
\end{aligned}
$$

Next, the 10 customers (i.e., the experts of the problem) classify the 5 interior designs (i.e., the alternatives of the problem).

Table 5 shows the importance class of each respondent and the relevant preference rankings. In general, the respondents could not be divided uniformly in the importance classes. In our case study we have 2 experts in the class A, 2 experts in the class B, and 3 experts respectively in the classes $\mathrm{C}$ and $\mathrm{D}$.

We now consider the three paradigms introduced in Section 3. 


\begin{tabular}{ccll} 
Expert & Importance class & Preference ranking \\
\hline$e_{1}$ & $\mathrm{~A}$ & $R_{1}:$ & $x_{1} \succ x_{3} \succ\left(x_{2} \sim x_{5}\right) \succ x_{4}$ \\
$e_{2}$ & $\mathrm{~B}$ & $R_{2}:$ & $x_{2} \succ x_{5} \succ\left(x_{1} \sim x_{3} \sim x_{4}\right)$ \\
$e_{3}$ & $\mathrm{C}$ & $R_{3}:$ & $x_{1} \succ\left(x_{2} \sim x_{5}\right) \succ x_{4} \succ x_{3}$ \\
$e_{4}$ & $\mathrm{~B}$ & $R_{4}:$ & $x_{5} \succ x_{2} \succ x_{3} \succ\left(x_{1} \sim x_{4}\right)$ \\
$e_{5}$ & $\mathrm{C}$ & $R_{5}:$ & $x_{5} \succ x_{2} \succ\left(x_{1} \sim x_{3}\right) \succ x_{4}$ \\
$e_{6}$ & $\mathrm{D}$ & $R_{6}:$ & $\left(x_{1} \sim x_{2}\right) \succ x_{5} \succ\left(x_{3} \sim x_{4}\right)$ \\
$e_{7}$ & $\mathrm{~A}$ & $R_{7}:$ & $x_{1} \succ\left(x_{2} \sim x_{3} \sim x_{5}\right) \succ x_{4}$ \\
$e_{8}$ & $\mathrm{D}$ & $R_{8}:$ & $x_{3} \succ\left(x_{1} \sim x_{2}\right) \succ x_{5} \succ x_{4}$ \\
$e_{9}$ & $\mathrm{D}$ & $R_{9}:$ & $x_{5} \succ\left(x_{1} \sim x_{2}\right) \succ\left(x_{3} \sim x_{4}\right)$ \\
$e_{10}$ & $\mathrm{C}$ & $R_{10}:$ & $x_{2} \succ x_{5} \succ x_{3} \succ x_{1} \succ x_{4}$
\end{tabular}

Table 5: Preference rankings related to the ten experts surveyed.

\subsection{Assigning a numerical weight to each expert}

Under the approach of Subsection 3.1, consider the following percentages of importance to A, B, C and D: $40 \%, 35 \%, 15 \%$ and $10 \%$, respectively. Then, $\left(t_{1}, t_{2}, t_{3}, t_{4}, t_{5}, t_{6}, t_{7}, t_{8}, t_{9}, t_{10}\right)=(8,7,3,7,3,2,8,2,2,3)$.

Thus, we have the following profile:

$$
\begin{aligned}
& (\overbrace{R_{1}, \ldots, R_{1}}^{8}, \overbrace{R_{2}, \ldots, R_{2}}^{7}, \overbrace{R_{3}, \ldots, R_{3}}^{3}, \overbrace{R_{4}, \ldots, R_{4}}^{3}, \overbrace{R_{5}, \ldots, R_{5}}^{3}, \\
& \overbrace{R_{6}, \ldots, R_{6}}^{2}, \overbrace{R_{7}, \ldots, R_{7}}^{8}, \overbrace{R_{8}, \ldots, R_{8}}^{3}, \overbrace{R_{9}, \ldots, R_{9}}^{2}, \overbrace{R_{10}, \ldots, R_{10}}^{2}) \in W(X)^{45} .
\end{aligned}
$$

If we apply the Borda rule to this profile, we obtain the scores included in Table 6. Since $B^{*}\left(x_{i}\right)=\sum_{k=1}^{10} t_{k} \cdot B_{k}\left(x_{i}\right)$ and $B^{*}\left(x_{2}\right)=122.5>B^{*}\left(x_{5}\right)=$ $119.5>B^{*}\left(x_{1}\right)=111>B^{*}\left(x_{3}\right)=81.5>B^{*}\left(x_{4}\right)=15.5$, the final ranking of the alternatives is $x_{2} \succ x_{5} \succ x_{1} \succ x_{3} \succ x_{4}$.

\subsection{Hierarchy of experts}

As anticipated in Subsection 3.2, we can tackle the problem in two ways:

1. Direct method: adopting the YA algorithm or similar variants. In this specific case study we adopt the approach proposed by Franceschini et al. $[15,18]$ as a variant of the YA algorithm. The method is organized in three steps:

(a) Construction and reorganization of the expert preference vectors.

(b) Definition of the reading sequence. 


\begin{tabular}{crrrrrr} 
Alternatives & $x_{1}$ & $x_{2}$ & $x_{3}$ & $x_{4}$ & $x_{5}$ \\
\hline$t_{1} \cdot B_{1}\left(x_{i}\right)$ & $8 \cdot 4=32$ & $8 \cdot 1.5=12$ & $8 \cdot 3=24$ & $8 \cdot 0=0$ & $8 \cdot 1.5=12$ \\
$t_{2} \cdot B_{2}\left(x_{i}\right)$ & $7 \cdot 1=7$ & $7 \cdot 4=28$ & $7 \cdot 1=7$ & $7 \cdot 1=7$ & $7 \cdot 3=21$ \\
$t_{3} \cdot B_{3}\left(x_{i}\right)$ & $3 \cdot 4=12$ & $3 \cdot 2.5=7.5$ & $3 \cdot 0=0$ & $3 \cdot 1=3$ & $3 \cdot 2.5=7.5$ \\
$t_{4} \cdot B_{4}\left(x_{i}\right)$ & $7 \cdot 0.5=3.5$ & $7 \cdot 3=21$ & $7 \cdot 2=14$ & $7 \cdot 0.5=3.5$ & $7 \cdot 4=28$ \\
$t_{5} \cdot B_{5}\left(x_{i}\right)$ & $3 \cdot 1.5=4.5$ & $3 \cdot 3=9$ & $3 \cdot 1.5=4.5$ & $3 \cdot 0=0$ & $3 \cdot 4=12$ \\
$t_{6} \cdot B_{6}\left(x_{i}\right)$ & $2 \cdot 3.5=7$ & $2 \cdot 3.5=7$ & $2 \cdot 0.5=1$ & $2 \cdot 0.5=1$ & $2 \cdot 2=4$ \\
$t_{7} \cdot B_{7}\left(x_{i}\right)$ & $8 \cdot 4=32$ & $8 \cdot 2=16$ & $8 \cdot 2=16$ & $8 \cdot 0=0$ & $8 \cdot 2=16$ \\
$t_{8} \cdot B_{8}\left(x_{i}\right)$ & $2 \cdot 2.5=5$ & $2 \cdot 2.5=5$ & $2 \cdot 4=8$ & $2 \cdot 0=0$ & $2 \cdot 1=2$ \\
$t_{9} \cdot B_{9}\left(x_{i}\right)$ & $2 \cdot 2.5=5$ & $2 \cdot 2.5=5$ & $2 \cdot 0.5=1$ & $2 \cdot 0.5=1$ & $2 \cdot 4=8$ \\
$t_{10} \cdot B_{10}\left(x_{i}\right)$ & $3 \cdot 1=3$ & $3 \cdot 4=12$ & $3 \cdot 2=6$ & $3 \cdot 0=0$ & $3 \cdot 3=9$ \\
10 & & & & & \\
$\sum_{k=1} t_{k} \cdot B_{k}\left(x_{i}\right)$ & 111 & 122.5 & 81.5 & & \\
& & & & & 119.5
\end{tabular}

Table 6: Scores.

(c) Generation of the fused ranking.

Step (a). Based on the different classes we have the following hierarchy among experts:

$$
\left(e_{1} \sim e_{7}\right) \succ\left(e_{2} \sim e_{4}\right) \succ\left(e_{3} \sim e_{5} \sim e_{10}\right) \succ\left(e_{6} \sim e_{8} \sim e_{9}\right) .
$$

According to the four classes of importance, the preference rankings of Table 5 are reorganized in Table 7 . Classes are strictly decreasing in terms of importance. Each cell element originates from the level-by-level union of the preferences related to each single expert.

\begin{tabular}{ccccc} 
Importance class & $\mathrm{A}$ & $\mathrm{B}$ & $\mathrm{C}$ & $\mathrm{D}$ \\
Experts & $\left\{e_{1}, e_{7}\right\}$ & $\left\{e_{2}, e_{4}\right\}$ & $\left\{e_{3}, e_{5}, e_{10}\right\}$ & $\left\{e_{6}, e_{8}, e_{9}\right\}$ \\
\hline Reorganized & $x_{1} x_{1}$ & $x_{2} x_{5}$ & $x_{1} x_{2} x_{5}$ & $x_{1} x_{2} x_{3} x_{5}$ \\
preferences & $x_{2} x_{2} x_{3} x_{5}$ & $x_{2} x_{5}$ & $x_{2} x_{2} x_{5} x_{5}$ & $x_{1} x_{1} x_{2} x_{2} x_{5}$ \\
& $x_{2} x_{4} x_{5}$ & $x_{1} x_{3} x_{3} x_{4}$ & $x_{1} x_{3} x_{3} x_{4}$ & $x_{3} x_{3} x_{4} x_{4} x_{5}$ \\
& $x_{4}$ & $x_{1} x_{4}$ & $x_{1} x_{3} x_{4}$ & $x_{4}$ \\
& & & $x_{4}$ &
\end{tabular}

Table 7: Reorganized preferences according to the four classes of experts.

Step (b). The second step of the method concerns the construction of the reading sequence. The reading sequence represents the ordered path followed by the algorithm to allocate the alternative positions (see Table 
8). The logic of the sequence is to read the most preferred alternative first (Franceschini et al. [15]).

\begin{tabular}{ccccc}
$\begin{array}{c}\text { Importance class } \\
\text { Experts }\end{array}$ & $\mathrm{A}$ & $\mathrm{B}$ & $\mathrm{C}$ & $\mathrm{D}$ \\
& $\left\{e_{1}, e_{7}\right\}$ & $\left\{e_{2}, e_{4}\right\}$ & $\left\{e_{3}, e_{5}, e_{10}\right\}$ & $\left\{e_{6}, e_{8}, e_{9}\right\}$ \\
\hline 1 & 2 & 3 & 4 \\
5 & 6 & 7 & 8 \\
9 & 10 & 11 & 12 \\
13 & 14 & 15 & 16 \\
17 & 18 & 19 & 20
\end{tabular}

Table 8: Reading sequence number $(S)$ related to the reorganized vectors in Table 7.

Step (c). This last step generates the final fused ranking. A step-by-step application of the Ordinal Prioritization Method is illustrated in Table 9. A detailed description of the method is reported in Franceschini et al. [15]. Data is related to the example of Tables 7 and 8.

The first three columns are related to the reading sequence: $S$ is the sequence number, $j$ denotes the importance class selected, while the column Element (I) is the set of alternatives taken, step by step, from the table of the reorganized preferences (Table 7). The subsequent columns refer to the construction of the total ranking. $E$ is the set of alternatives included in the gradual ranking and $R$ is the set of alternatives not yet included in the gradual ranking (residual elements).

We remark that an alternative is added to the total ranking only when the number of occurrences is greater than or equal to $T_{k}$ (occurrence threshold). $T_{k}$ is defined by the algorithm designer. It is worth noting that greater values of $T_{k}$ assign less significance to the ranking of experts. In this case study we fix $T_{k}=3$ for all the alternatives. By this approach the final ranking of the alternatives is: $x_{1} \succ\left(x_{2} \sim x_{5}\right) \succ x_{3} \succ x_{4}$.

2. We can generate an "artificial" weighting vector based, for example, on the Borda scores obtained by the experts, coming back again to Subsection 3.1

Taking into account Eq. (1), we have

$$
\begin{aligned}
& B\left(e_{1}\right)=B\left(e_{7}\right)=8.5, B\left(e_{2}\right)=B\left(e_{4}\right)=6.5 \\
& B\left(e_{3}\right)=B\left(e_{5}\right)=B\left(e_{10}\right)=4, B\left(e_{6}\right)=B\left(e_{8}\right)=B\left(e_{9}\right)=1 .
\end{aligned}
$$

Hence, $t_{1}=t_{7}=17, t_{2}=t_{4}=13, t_{3}=t_{5}=t_{10}=8, t_{6}=t_{8}=t_{9}=2$. Consequently, $R_{1}, R_{2}, R_{3}, R_{4}, R_{5}, R_{6}, R_{7}, R_{8}, R_{9}$ and $R_{10}$ should be replicated $17,13,8,13,8,2,17,2,2$ and 8 times, respectively. 


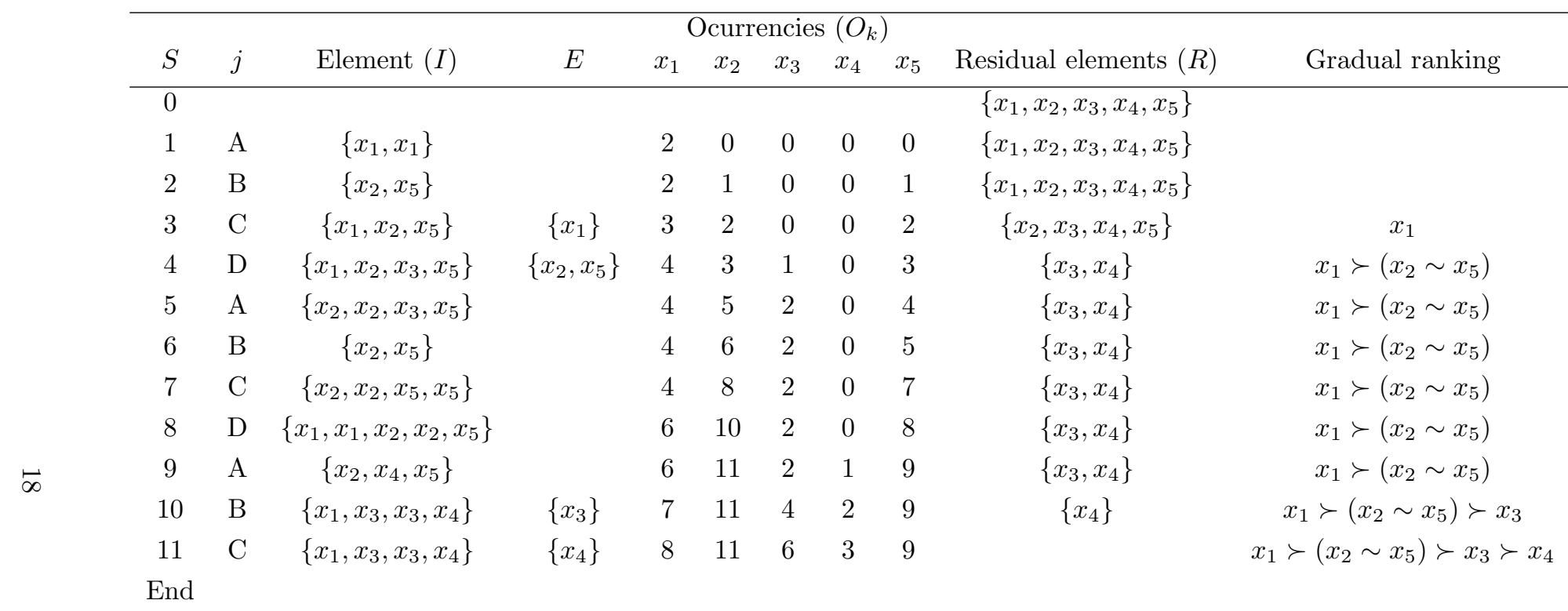

Table 9: Step-by-step application of the Ordinal Prioritization Method (Franceschini et al. [15]). 
Then, we have the following profile:

$$
\begin{aligned}
& (\overbrace{R_{1}, \ldots, R_{1}}^{17}, \overbrace{R_{2}, \ldots, R_{2}}^{13}, \overbrace{R_{3}, \ldots, R_{3}}^{8}, \overbrace{R_{4}, \ldots, R_{4}}^{13}, \overbrace{R_{5}, \ldots, R_{5}}^{8}, \overbrace{R_{6}, \ldots, R_{6}}^{2}, \\
& \overbrace{R_{7}, \ldots, R_{7}}^{17}, \overbrace{R_{8}, \ldots, R_{8}}^{2}, \overbrace{R_{9}, \ldots, R_{9}}^{2}, \overbrace{R_{10}, \ldots, R_{10}}^{8}) \in W(X)^{90} .
\end{aligned}
$$

If we apply the Borda rule to this profile, we obtain the following total scores for $x_{1}, x_{2}, x_{3}, x_{4}$ and $x_{5}$, respectively: 224.5, 243.5, 162, 29.5 and 240.5. Then, the final ranking of the alternatives is $x_{2} \succ x_{5} \succ x_{1} \succ$ $x_{3} \succ x_{4}$.

\subsection{Ordinal proximity measures}

Under the approach of Subsection 3.3, we will consider three different OPMs. Once the number of replications of each weak order are obtained, following the procedure illustrated in Example 3, we apply the Borda rule to the corresponding profiles.

(a) With the OPM with associated proximity matrix

$$
A_{323}=\left(\begin{array}{cccc}
\delta_{1} & \delta_{3} & \delta_{4} & \delta_{5} \\
& \delta_{1} & \delta_{2} & \delta_{4} \\
& & \delta_{1} & \delta_{3} \\
& & & \delta_{1}
\end{array}\right),
$$

that can be visualized in Fig. 4, the final ranking of the alternatives is: $x_{1} \succ x_{2} \succ x_{5} \succ x_{3} \succ x_{4}$.

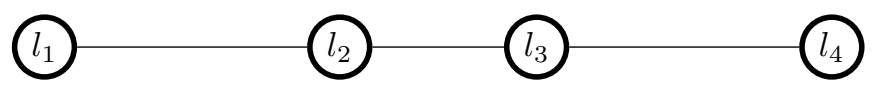

Figure 4: Ordinal proximity measure with associated matrix $A_{323}$.

(b) With the OPM with associated proximity matrix

$$
A_{232}=\left(\begin{array}{cccc}
\delta_{1} & \delta_{2} & \delta_{4} & \delta_{5} \\
& \delta_{1} & \delta_{3} & \delta_{4} \\
& & \delta_{1} & \delta_{2} \\
& & & \delta_{1}
\end{array}\right),
$$

that can be visualized in Fig. 2, the final ranking of the alternatives is: $x_{2} \succ x_{5} \succ x_{1} \succ x_{3} \succ x_{4}$. 
(c) With the OPM with associated proximity matrix

$$
A_{423}=\left(\begin{array}{cccc}
\delta_{1} & \delta_{4} & \delta_{6} & \delta_{7} \\
& \delta_{1} & \delta_{2} & \delta_{5} \\
& & \delta_{1} & \delta_{3} \\
& & & \delta_{1}
\end{array}\right),
$$

that can be visualized in Fig. 5, the final ranking of the alternatives is: $x_{1} \succ x_{2} \succ x_{5} \succ x_{3} \succ x_{4}$.

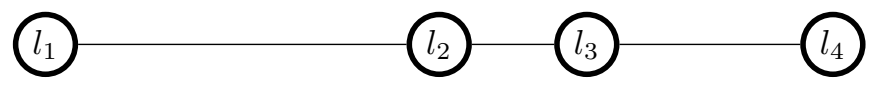

Figure 5: Ordinal proximity measure with associated matrix $A_{423}$.

Taking into account the opinions of the ten customers on the five interior designs of a new car included in Table 5, the outcomes obtained under the approaches introduced in Subsections 3.1, 3.2 and 3.3, developed for the case study in Subsections 4.1, 4.2 and 4.3, respectively, are summarized in Table 10.

\begin{tabular}{cccl}
$\begin{array}{c}\text { Approach } \\
\text { Subsection }\end{array}$ & $\begin{array}{c}\text { Case study } \\
\text { Subsection }\end{array}$ & Subcase & Preference ranking \\
\hline 3.1 & 4.1 & & $x_{2} \succ x_{5} \succ x_{1} \succ x_{3} \succ x_{4}$ \\
3.2 & 4.2 & 1 & $x_{1} \succ\left(x_{2} \sim x_{5}\right) \succ x_{3} \succ x_{4}$ \\
3.2 & 4.2 & 2 & $x_{2} \succ x_{5} \succ x_{1} \succ x_{3} \succ x_{4}$ \\
3.3 & 4.3 & $A_{323}$ & $x_{1} \succ x_{2} \succ x_{5} \succ x_{3} \succ x_{4}$ \\
3.3 & 4.3 & $A_{232}$ & $x_{2} \succ x_{5} \succ x_{1} \succ x_{3} \succ x_{4}$ \\
3.3 & 4.3 & $A_{423}$ & $x_{1} \succ x_{2} \succ x_{5} \succ x_{3} \succ x_{4}$
\end{tabular}

Table 10: Summary.

The winner is $x_{1}$ or $x_{2}$, depending on the case, but when $x_{2}$ is the winner, $x_{1}$ is always in the third position. The fourth and the fifth positions are always for $x_{3}$ and $x_{4}$, respectively.

Taking into account the six cases considered, $x_{1}, x_{2}, x_{3}, x_{4}$ and $x_{5}$ have average positions $2,1.58,3,4$ and 2.42 , respectively. Thus, on average, the final ranking is $x_{2} \succ x_{1} \succ x_{5} \succ x_{3} \succ x_{4}$, that it does not coincide with any of the outcomes obtained in the six cases. Notice that this ranking is the same than the one obtained when applying the Borda rule to the six preference rankings of Table 10. This is due to the fact that the Borda rule ranks the alternatives according to their average positions.

\section{Conclusions}

The proposed method allow to aggregate multi-experts rankings of different alternatives into a single fused ranking according to different semi-democratic 
paradigms: (i) the importance of experts is directly reported by means of a weighing vector; (ii) the importance of experts is expressed by a weak order on the set of expert; (iii) the importance of experts is described by a weak order with ordinal proximity measures on the set of expert. The three paradigms can be applied in different decision-making situations, where some experts perform multiple assignments.

The results obtained in the case study highlight the following aspects:

- Different methods lead to different rankings of the alternatives, even if sometimes they appear to coincide.

- There is a general agreement between the methods for top and bottom positions in the rankings.

- The use of one method or another depends on the quality of information available from the different semi-democratic decision making contexts.

It is important to mention some advantages of the methods proposed in this paper with respect to other proposals:

- Their simplicity and the greater adherence of data properties to real situations.

- Weights are treated in a purely ordinal way by replicating experts' opinions according to the proportions between weights.

- The use of minimal structures for representing hierarchies on the set of experts (weak orders and ordinal proximity measures). For instance, taking a particular membership function is a much stronger hypothesis than considering a weak order between experts.

The main contribution of this paper is to provide a general overview of the state-of-art of the methods able to tackle decision-making problems in semidemocratic contexts. In our analysis it was assumed that the preference rankings of experts are complete; i.e., all experts are able to rank all the alternatives of interest, without omitting any of them. The analysis does not consider the (possible) uncertainty in expert rankings, and/or preference rankings with incomparability between some alternatives.

Regarding the future, we plan to extend the analysis to situations where experts are not able to provide complete weak rankings, but only partial preference rankings, uncertainty rankings or even rankings with some forms of incomparability between alternatives to be evaluated.

\section{Acknowledgments.}

Financial support from Spanish Ministerio de Economía y Competitividad (project ECO2016-77900-P) and ERDF is acknowledged. 


\section{References}

[1] de Andrés, R., García-Lapresta, J.L., Martínez, L.: A multi-granular linguistic model for management decision-making in performance appraisal. Soft Computing 14(1), pp. 21-34, 2010.

[2] Arrow, K.J.: Social Choice and Individual Values, 3rd edition. Yale University Press, New Haven, 2012.

[3] Arrow, K.J., Raynaud, H.: Social Choice and Multicriterion Decision Making. MIT Press, Cambridge, 1986.

[4] Black, D.: Partial justification of the Borda count. Public Choice 28, pp. $1-16,1976$.

[5] Borda, J.C. de: Mémoire sur les élections au scrutin. Historie de l'Academie Royale des Sciences, Paris, 1784.

[6] Brans, J.P., Mareschal, B.: Promethee methods. In: S. Greco, M. Ehrgott, J.R. Figueira (Eds.), Multiple Criteria Decision Analysis: State of the Art Surveys. International Series in Operations Research \& Management Science 233, Springer, pp. 187-219, 2016.

[7] Chebotarev, P.Y., Shamis, E.: Characterizations of scoring methods for preference aggregation. Annals of Operations Research 80, pp. 299-332, 1998.

[8] Chen, S., Liu, J., Wang, H., Augusto, J.C.: Ordering based decision making - A survey. Information Fusion 14, pp. 521-531, 2013.

[9] Colomer, J.M. (Ed.): The Handbook of Electoral System Choice. Palgrave Macmillan, New York, 2004.

[10] Cook, W.D.: Distance-based and ad hoc consensus models in ordinal preference ranking. European Journal of Operational Research 172, pp. 369-385, 2006.

[11] Dym, C.L., Wood, W.H., Scott, M.J.: Rank ordering engineering designs: pairwise comparison charts and Borda counts. Research in Engineering Design 13, pp. 236-242, 2002.

[12] Dubois, D., Godo, L., Prade, H.: Weighted logics for artificial intelligence - an introductory discussion. International Journal of Approximate Reasoning 55, pp. 1819-1829, 2014.

[13] Fishburn, P.C.: The Theory of Social Choice. Princeton University Press, Princeton, 1973.

[14] Franceschini, F., Galetto, M., Maisano, D.: Management by Measurement: Designing Key Indicators and Performance Measurement Systems. Springer, Berlin, 2007. 
[15] Franceschini, F., Galetto, M., Maisano, D., Mastrogiacomo, L.: Prioritisation of engineering characteristics in QFD in the case of customer requirements orderings. International Journal of Production Research 53(13), pp. 3975-3988, 2015.

[16] Franceschini, F., Maisano, D.: Checking the consistency of the solution in ordinal semi-democratic decision-making problems. Omega 57(1), pp. 188-195, 2015.

[17] Franceschini, F., Maisano, D.: Consistency analysis in quality classification problems with multiple rank-ordered agents. Quality Engineering 29(4), pp. 672-689, 2017.

[18] Franceschini, F., Maisano, D., Mastrogiacomo, L.: A new proposal for fusing individual preference orderings by rank-ordered agents: A generalization of the Yager's algorithm. European Journal of Operational Research 249, pp. 209-223, 2016.

[19] Franssen, M.: Arrow's theorem, multi-criteria decision problems and multiattribute preferences in engineering design. Research in Engineering Design 16(1-2), pp. 42-56, 2005.

[20] García-Lapresta, J.L., González del Pozo, R.: An ordinal multi-criteria decision-making procedure in the context of uniform qualitative scales. In: M. Collan, J. Kacprzyk (Eds.), Soft Computing Applications for Group Decision-making and Consensus Modeling, Studies in Fuzziness and Soft Computing 357, Springer, pp. 297-304, 2018.

[21] García-Lapresta, J.L., González del Pozo, R., Pérez-Román, D.: Metrizable ordinal proximity measures and their aggregation. Information Sciences 448-449, pp. 149-163, 2018.

[22] García-Lapresta, J.L., Pérez-Román, D.: Ordinal proximity measures in the context of unbalanced qualitative scales and some applications to consensus and clustering. Applied Soft Computing 35, pp. 864-872, 2015.

[23] Greco, S., Ehrgott, M., Figueira, J.R. (Eds.): Multiple Criteria Decision Analysis: State of the Art Surveys. International Series in Operations Research \& Management Science 233, Springer, 2016.

[24] Griffin, A., Hauser, J.R.: The voice of the customer. Marketing Science 12, pp. 1-27, 1993.

[25] Herrera-Viedma, E., Cabrerizo, F.J., Kacprzyk, J., Pedrycz, W.: A review of soft consensus models in a fuzzy environment. Information Fusion 17, pp. 4-13, 2014.

[26] Hu, J., Pan, L., Yang, Y., Chen, H.: A group medical diagnosis model based on intuitionistic fuzzy soft sets. Applied Soft Computing 77, pp. 453-466, 2019. 
[27] Jacobs, J.F., van de Poel, I., Osseweijer, P.: Clarifying the debate on selection methods for engineering: Arrow's impossibility theorem, design performances, and information basis. Research in Engineering Design 25(1), pp. 3-10, 2014.

[28] Keeney, R.L.: The foundations of collaborative group decisions. International Journal of Collaborative Engineering 1, pp. 4-18, 2009.

[29] Ladha, K., Miller, G., Oppenheimer, J.: Information aggregation by majority rule: Theory and experiments.

http://www.gvptsites.umd.edu/oppenheimer/research/jury.pdf, 2003.

[30] Martel, J.B., Ben Khélifa, S.: Deux propositions d'aide multicritère à la décision de groupe. In: F. Ben Abdelaziz, M. Haouari, K. Mellouli (Eds.), Optimisation et Décision, Centre de Publication Universitaire, pp. 213-228, 2000 .

[31] McComb, C., Goucher-Lambert, K., Cagan, J.: Impossible by design? Fairness, strategy and Arrow's impossibility theorem. Design Science 3, E2, DOI:10.1017/dsj.2017.1, 2017.

[32] Mishra, A.R., Rani, P.: Interval-valued intuitionistic fuzzy WASPAS method: Application in reservoir flood control management policy. Group Decision and Negotiation 27, pp. 1047-1078, 2018.

[33] Møller, J., Skaaning, S.E.: Beyond the radial delusion: Conceptualizing and measuring democracy and non-democracy. International Political Science Review 31(3), pp. 261-283, 2010 .

[34] Nurmi, H.: On the relevance of theoretical results to voting system choice. In: D.S. Felsenthal, M. Machover (Eds.), Electoral Systems: Studies in Choice and Welfare, Springer, pp. 255-274, 2012.

[35] Önüt, S., Kara, S.S., Efendigil, T.: A hybrid fuzzy MCDM approach to machine tool selection. Journal of Intelligent Manufacturing 19(4), pp. 443453, 2008.

[36] Saari D.G.: Decisions and Elections: Explaining the Unexpected. Cambridge University Press, Cambridge, 2001.

[37] Saaty, T.L.: The Analytic Hierarchy Process. McGraw-Hill, New York, 1980.

[38] See, T.K., Lewis, K.: A formal approach to handling conflicts in multiattribute group decision making. Journal of Mechanical Design 128(4), pp. 678-688, 2006.

[39] Sen, A.: Poverty: an ordinal approach to measurement. Econometrica 44, pp. 219-231, 1976. 
[40] Ureña, R., Kou, G., Dong, Y., Chiclana, F., Herrera-Viedma, E.: A review on trust propagation and opinion dynamics in social networks and group decision making frameworks. Information Sciences 478, pp. 461-475, 2019.

[41] Wang, J.Q.: Fusion of multiagent preference orderings with information on agent's importance being incomplete certain. Journal of Systems Engineering and Electronics 18, pp. 801-805, 2007.

[42] Wang, B., Liang, J., Qian, Y.: Determining decision makers' weights in group ranking: a granular computing method. International Journal of Machine Learning and Cybernetics 6, pp. 511-521, 2014.

[43] Wu, Y., Li ,C.C., Chen, X., Dong, Y.: Group decision making based on linguistic distributions and hesitant assessments: Maximizing the support degree with an accuracy constraint. Information Fusion 41, pp. 151-160, 2018.

[44] Yager, R.R.: Intelligent agents for World Wide Web advertising decisions. International Journal of Intelligent Systems 12, pp. 379-390, 1997.

[45] Yager, R.R.: Fusion of multi-agent preference orderings. Fuzzy Sets and Systems 117, pp. 1-12, 2001.

[46] Yu, W., Zhang, Z., Zhong, Q., Sun, L.: Extended TODIM for multi-criteria group decision making based on unbalanced hesitant fuzzy linguistic term sets. Computers \& Industrial Engineering 114, pp. 316-328, 2017.

[47] Yue, Z.L.: Deriving decision maker's weights based on distance measure for interval-valued intuitionistic fuzzy group decision making. Expert Systems with Applications 38(9), pp. 11665-11670, 2011.

[48] Zhang, Z., Guo, C.: Deriving priority weights from intuitionistic multiplicative preference relations under group decision-making settings. Journal of the Operational Research Society 68(12), pp. 1582-1599, 2017.

[49] Zhang, Z., Guo, C., Martínez, L.: Managing multigranular linguistic distribution assessments in large-scale multiattribute group decision making. IEEE Transactions on Systems, Man, and Cybernetics: Systems 47(11), pp. 3063-3076, 2017. 